\title{
BMJ Open Integrating social support into interventions among the elderly in nursing homes: a scoping review protocol
}

\author{
Daniel Behrendt (D), Marielle Schirmer, Vanessa Wendschuh, \\ Chommanard Sumngern
}

To cite: Behrendt $D$,

Schirmer M, Wendschuh V, et al. Integrating social support into interventions among the elderly in nursing homes: a scoping review protocol. BMJ Open 2022;12:e055692. doi:10.1136/ bmjopen-2021-055692

- Prepublication history and additional supplemental material for this paper are available online. To view these files, please visit the journal online (http://dx.doi.org/10.1136/ bmjopen-2021-055692).

Received 20 July 2021 Accepted 12 January 2022

Check for updates

(c) Author(s) (or their employer(s)) 2022. Re-use permitted under CC BY-NC. No commercial re-use. See rights and permissions. Published by BMJ.

Nursing Department, Städtisches Klinikum Dessau, Dessau, Germany

Correspondence to Mr Daniel Behrendt; daniel.behrendt@klinikumdessau.de

\section{ABSTRACT}

Introduction The instituted elderly in nursing home need professional support targeting to maintain their daily activities and quality of life. Social support affects the health of elderly through its influence as a stress buffering or main effects on emotions, cognitions and behaviour that improve health outcomes. Understanding and identifying available interventions for the elderly in the literature related to integrating social support into interventions will be benefits to guide future practice, research and policy. Methods and analysis $A$ scoping review designed by Arksey and O'Malley has been used in this study. Key words, inclusion and exclusion criteria were elaborated to search the primary articles that published in English from 2010 to 2021 mainly from PubMed, Science Direct, Public Library of Science, SocioHub, Wiley Online Library and PsycINF0 databases targeting to reach the selected articles and combined the results with reference lists and hand searches. The Mixed Methods Appraisal Tool version 2018 will be used to identify the quality of the studies. Authors developed the Data Extraction Form for data extraction and analysis. The Preferred Reporting Items for Systematic Reviews and Meta-analyses extension for Scoping Reviews tool will be employed for reporting guideline.

Ethics and dissemination Authors forecast to obtain relevant studies reporting integrating specific dimensions of supportive functions into interventions for the elderly in nursing home. This finding will benefit in quality improvement of supportive interventions in nursing home and to continue the further experimental study. The findings will be disseminated via electronic and hard copy through peer-reviewed publications, conference presentations and internal organisation meeting.

\section{INTRODUCTION}

Ageing is a global phenomenon where older people resulting from multiple loses and changes caused by ageing process as well as physical, psychological and pathological conditions. Generally, elderly prefer to live at home with their family, but they have been often instituted because of health problems and/or chronic illness. The instituted elderly
Strengths and limitations of this study

- Social support concept will be identified in social care services in nursing home.

- This study will be the first report about dimensions of supportive function of the interventions in nursing home.

The quality of the studies will be reported.

- The Preferred Reporting Items for Systematic Reviews and Meta-Analyses extension for Scoping Reviews tool has been employed.

- Searching the relevant articles only in English and only primary research may be study limitations.

need more specific help and social care from formal caregivers for their personal strength and ability to maintain their quality of life (QOL). ${ }^{1}$ Moreover, elderly need high budgets for treatments and care to perform their daily activities. $^{23}$

WHO stated on the strategic objectives for providing long-term care (LTC) that systems are needed in all countries to meet the needs of older people and to benefit beyond enabling care-dependent older people to do what they value and to live lives of dignity. ${ }^{4}$ LTC is formally an important part of social care and an effective policy should provide support for a range of options, including home care services, assisted-living facilities and nursing home. ${ }^{5}$

Nursing home refers to a residential institutional setting that provides LTC with 24-hour functional support for people who need assistance with several activities of daily living (ADLs) and instrumental ADLs. ${ }^{5}{ }^{6}$ Generally, nursing home supports care for elderly who require nursing cares which were hard to provide by family members, helpers and specialists in home settings. ${ }^{7}$ There are various reasons that elderly got admitted in nursing home such as suffering from chronic 
diseases, limited to extensive ADLs, mobility impairment, living alone and cognitive disturbance. ${ }^{89}$ In addition, living in nursing home may also cause negative effects to the elderly. A study in Norway reported that suffering was prominent among the elderly in nursing home because of painful experiences during life, mental health, bodily pain, emotional role limitations and lack of social relationships. ${ }^{10}$

Presently, psychosocial impacts of corona pandemic from social isolation have been worldwide concerned as having seriously harmful consequences especially on cognitive impairment residents including increased depression, loneliness and worsening dementia. ${ }^{11}$ Social isolation and particularly recent isolation due to pandemic together with poor social support are ones of the risk factors for suicide in older people. ${ }^{5}$ Multiple loses in their age along with the transition to LTC can be stressful life event that may make older people decrease their QOL. ${ }^{12}$

Social services and health outcomes have been enacted in policies to increase life expectancy age and to promote healthy life expectancy targeting to improve the QOL of the senior citizen and these services have been depicted the positive development. ${ }^{2} 1314$ A security from healthcare insurance related to policy together with a better individual financial literacy and an effective mean of supportive intervention from healthcare provider are important determinants influencing QOL among the elderly. ${ }^{15}$ There are limitations of social services in nursing homes. The services may not cover or not match with the individual needs because of the differences between the perspectives about care needs of residents and staff. ${ }^{16}$ Healthcare specialists such as gerontologist and social gerontologist have been concerned as persons who can provide the intervention for the instituted elderly. ${ }^{17-19}$ Providing a decent care for the elderly living in nursing home depends on not only from the intervention design itself, but also from providers. However, healthcare provider shortage is a typical problem worldwide, thus an increasing of formal caregivers has been issued as one of the necessary strategies in public health sectors. ${ }^{20-22}$

Social support refers to relationships among people that provide not only material help and emotional assurance, but also the sense that one is a continuing object of concern on the part of other people. ${ }^{23}$ Based on theoretical and literatures, social support has been confirmed as one of the important determinants which affects to individuals health through its influence as a stress buffering or main effects on emotions, cognitions and behaviour that improve both of physical and psychological health outcomes. ${ }^{24-26}$ Uchino confirmed from the study in 2006 that social support affects to cardiovascular, neuroendocrine and immune function. ${ }^{27}$ Turner and Brown concluded their literature reviews related to social support and mental health; they found a clear inverse association between perceived social support and depression. ${ }^{28}$ This is in agreement with the study in Malaysian nursing homes during the COVID-19 pandemic, the authors indicated that the elderly with low perceived social support associated with depression. ${ }^{29}$ Also, the study of Wang et al found that individuals who obtained higher levels of social support might have more positive health outcomes including health status, role function and behaviours, psychosocial adjustment, adjustment of life, coping behaviour, health belief, health promotion behaviour, QOL, well-being and self-actualisation. ${ }^{30}$

Social support could also negatively impacts as a significant source of stress for unhealthy behaviours such as feeling of indebtedness or guilt to the support receivers. ${ }^{27} 31$ It is noteworthy that specific dimensions of supportive function referring to both of quantitative and qualitative aspects depict beneficial roles on health as personal resources in different stress life events. ${ }^{31}$ Veiel pointed out that types of available support should be included in the social support assessment. ${ }^{32}$ In addition, Wills and Shinar depicted the dimensions of available support or functional support relating to social support intervention in terms of emotional support, instrumental support, informational support, companionship support and validation which provide specific theoretically expected benefits for coping efforts. ${ }^{33}$

As known in literature studies, a trend towards a positive effect of social support and social interventions on social and health-related outcomes among older people in nursing homes has been receiving attention. ${ }^{34}$ Even though there were two review articles regarding to social support and interventions among the elderly living in nursing homes in terms of social interaction ${ }^{35}$ and social relation, ${ }^{34}$ none of the these studies clearly reported about dimensions of supportive function or type of social support, and supportive providers. Besides, there is no current or underway study on integrating social support into interventions among the elderly in nursing homes.

Generally, involvement in personally meaningful activity is a basic human drive that is essential to maintain physical and mental health, regardless of age or impairment. ${ }^{36}$ Theoretically, social support activities are important for the elderly as coping resources. ${ }^{24}$ Callaghan and Morrissey asserted that social support affects individual health in three ways by (1) regulating thoughts, feelings and behaviour as to promote health, (2) fostering an individual's sense of meaning in life and (3) facilitating health promoting behaviours. ${ }^{37}$ However, it is not easy to encourage the elderly who have psychological and physical health problems to identify the meaningful activities and to participate in activities. ${ }^{38}$ Consequently, evidencebased planning and well-prepared healthcare providers should be worthy strategies to elevate effective care covering the needs of the elderly living in nursing homes.

Scoping review is one type of review that can be used to map the key concepts that underpin a field of research, as well as to clarify working definitions, and/or the conceptual boundaries of a topic. ${ }^{39} 40$ The indications for this scoping review are to understand situations, to identify knowledge gaps and to clarify definitions in the literature related to integrating social support into interventions 
for the elderly living in nursing home. The findings from this review will be used to guide the policy and to develop proper social interventions for the elderly living in nursing home including identifying gaps in this subject and guiding us to design further study.

\section{Objectives}

The aims of this scoping review were to understand situations of providing social support into interventions among the elderly in nursing homes, to identify literature gaps in social support interventions, and to clarify as well as to map definitions of dimensions of supportive functions, outcomes, and providers with supportive interventions among the elderly in nursing homes.

\section{METHODS AND ANALYSIS}

A scoping review designed by Arksey and O'Malley in the year $2005^{39}$ was used in this study. The five stages of review includes (1) identifying the research question, (2) identifying relevant studies, (3) study selection, (4) charting the data and (5) collating, summarising and reporting the results. In case of necessary, Framework Optional Stage: consultation exercise will be performed. Following the recommendations from the studies of Lockwood et $a t^{41}$ and Daudt $e t a t^{2}$ authors will identify the quality of the studies.

\section{Stage 1: identifying the research question}

Scoping review questions include main question and subquestions as follows:

\section{Main question}

What has been studied so far about the providing social support into interventions among the elderly in nursing home?

\section{Subquestions}

1. What are interventions and outcomes related to social support?

2. Who are intervention providers in nursing home?

3. Which dimensions of supportive functions were integrated into interventions?

\section{Stage 2: identifying relevant studies}

Eligibility criteria

In this stage, inclusion and exclusion criteria will be set and used to identify the relevant studies. Moreover, authors will also select studies by reading titles and abstracts of articles published in English from 1 January 2010 to 31 March 2021, and full-text available. The Population, Concept, Context framework and types of sources were used to set the inclusion and exclusion criteria as follows:

\section{Participants}

Participants in selected records will be only elderly population (older adults, geriatrics, ageing, seniors, older people, and aged 60 years and older). The review will exclude studies that care recipient group were infant, child, childhood, children, adolescent, mother, parent, woman, man and homeless.

\section{Settings}

Studies took place only in nursing homes and care homes, will be selected. Settings including communitybased study, primary care, home(s), home care, day care centre(s), hospital(s), clinics, special clinics, emergency department and outpatient will be excluded.

\section{Types of sources}

Selected records will be primary studies and heterogeneous study designs including all types of qualitative, quantitative and mixed-methods studies. The review excludes all types of reviews, protocols, book chapters, opinion papers, editorial letters, guidelines and annual conference.

\section{Search strategy}

Authors will perform a scoping review on empirics of primary studies about social support and interventions which related to social support among the elderly living in nursing homes. The search strategy, including all identified keywords and index terms, will be adapted for each included database and/or information sources. Identifying the research questions, keywords derived from research questions including social support, intervention (activity and programme), outcomes and provider (staff), elderly and nursing home (care home) will be elaborated.

Taking into account the relevance of thousands of citations retrieved from bibliographic database searches, we agree with the mention of Bramer et al that welldeveloped bibliographic searches are generally created with thesaurus or index terms in combination with keywords found in the title and/or abstract fields of citation records. ${ }^{43}$ Searching the relevant articles using text words contained in the titles and/or abstracts will be performed mainly from available social science and medicine databases of the University Library including PubMed, Science Direct, Public Library of Science, SocioHub, Wiley Online Library and PsycINFO databases and hospital library services targeting to reach the selected articles (see online supplemental file 1).

\section{Stage 3: study selection}

All identified studies will be collated and uploaded into the reference management software 'Citavi V.6' and duplicates will be removed. Titles and abstracts will be independently screened concerning the inclusion and exclusion criteria by two authors (MS and CS). Then, two authors (VW and CS) will assess in detail and read carefully full text of articles to identify the articles from words and/or context of the studies which showing relation to the meaning of social support and the interpretation of dimensions of supportive function. Words and contexts of the studies related to social support functions based on social support concept by Wills and Shinar ${ }^{33}$ include emotional support (confidant support, esteem support, 


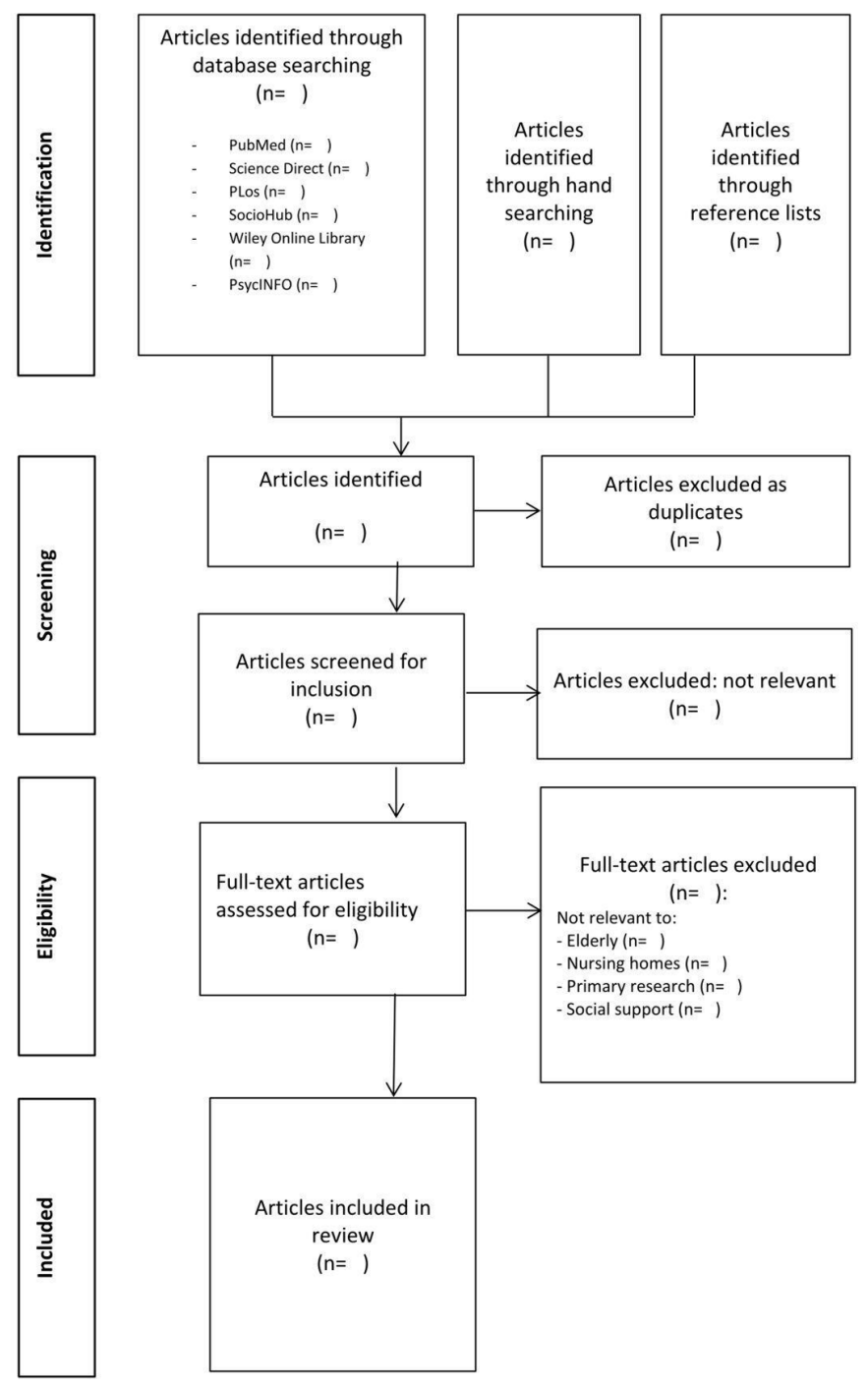

Figure 1 PRISMA flow diagram for study selection process. ${ }^{45}$ PLoS, Public Library of Science; PRISMA, Preferred Reporting Items for Systematic Reviews and Metaanalyses.

reassurance of worth, attachment, intimacy), instructional support (tangible support, practical support, behavioural assistant, material aid), informational support (advice/ guidance, problem solving), companionship support (belonging, socialising, integration and validation (feedback, social comparison). Besides, the reviewers will select the additional articles from hand searching and the reference lists of selected articles. Reasons for exclusion of evidence that do not meet the inclusion criteria will be recorded. Any disagreements that reviewers can not make clear decisions at each stage of selection process will be resolved through discussion of all authors (DB, MS, VW and CS). The searching results will be showed as flow diagram following a Preferred Reporting Items for Systematic Reviews and Meta-Analyses extension for Scoping Review (PRISMA-ScR) ${ }^{44}$ (see figure 1), which has been recommended by the Joanna Briggs Institute (JBI) as a part of methodological guidance for the conduct of scoping reviews. ${ }^{46} 47$
Quality of studies

The Mixed Methods Appraisal Tool version $2018^{48}$ will be used to identify the quality of the studies. This instrument has been proven useful in other mixed-methods systematic reviews encompassing a transparent and comparable approach to appraise studies with different designs. ${ }^{49} 50$ First, MS, VW and CS will perform the initial quality assessment and then it will be discussed in detail by all authors. As well, the quality of studies will be depicted in tabular form.

\section{Data extraction}

Data extraction form was developed based on JBI's manual for scoping reviews ${ }^{44}$ and will be used for data extraction. Primarily, MS, VW and CS will independently extract data, and data will be discussed again by all authors. The data extraction will include key information about authors (s), year of publication, country, objectives, study population, sample size and study methods including findings related to review questions: intervention design, intervention outcomes, supportive providers and dimensions of supportive function.

\section{Stage 4: charting the data}

Data charting will be performed (see table 1), and study characteristics will be extracted about author and year of publication, continent of study, types of study methods, total participants, chronological age of nursing home residents which will be grouped into young-old, old-old and extremely old. ${ }^{51}$ As well, the authors will categorise groups of nursing home residents into healthy residents and residents with health problems based on guidelines of integrated care for older people by $\mathrm{WHO}^{52}$ including mobility loss, undernutrition, vision impairment, hearing loss, cognitive impairment, depression symptoms, urinary incontinence, risk of falls and caregiver support. Concerning the interventions, the evidence will also be presented about length and frequency of interventions, outcomes, providers and dimensions of supportive function.

\section{Stage 5: collating, summarising and reporting the results}

Data analysis will be performed. A narrative summary about characteristics and descriptive data related to review objective and review questions will be described and depicted graphically or diagrammatic or tabular form.

\section{Data synthesis}

After extracting all data, we will perform the evidence synthesis. The findings will be discussed concerning the review questions covering characteristics of intervention and its outcome, supportive providers, and types of social support being integrated into interventions among the elderly in nursing home and particularly.

\section{Implications}

Limitations of this study will be discussed. Social support services are generally provided to meet the needs of the 
Table 1 Charting the data

Count

(no of

Scoping review details

studies, \%)

Year of publication
Continent of study
Study methods/designs
Qualitative research

Randomised controlled trials

Non-randomised studies

Quantitative descriptive studies

Mixed methods studies

Total participants

Age groups
Young-old (60-79)
Old-old (80-89)
Extremely old (90 and above)
Target groups of intervention received
residents
Healthy residents
Residents with health problems: mobility
loss, malnutrition, vision impairment, hearing
loss, cognitive impairment, depression,
urinary incontinence, risk of fall and elderly
caregiver
Interventions
Intervention design (type, duration,
frequency and modes of delivery)
Intervention outcomes
Supportive providers
Dimensions of supportive function

elderly who depend on others owing to the changes in physical and psychological domains caused by ageing process and pathology through its influence on emotions, cognitions and behaviours. All results will be discussed and will be used to classify the proper dimensions of supportive function integrating into the interventions for the elderly living in nursing homes, especially in and after a period of corona pandemic. In addition, gaps in this review topic will be identified and guide us to design further study and/or questions for future systematic review or experimental research.

\section{Ethics and dissemination}

Authors forecast to obtain relevant studies from literatures. This study was not performed for ethical approval. The PRISMA extension for Scoping Reviews (PRISMA-ScR) tool will be employed for reporting guideline. The results will be propagated via electronic and hard copy through peer-reviewed publications, conference presentations and internal organisation meeting. This finding will benefit in quality improvement to improve supportive interventions among the elderly in nursing home.

Acknowledgements The authors wish to thank Leipzig University Library for providing available useful information of databases and Library of Städtisches Klinikum Dessau for kindness collaboration. We are also grateful to Dr. Nilesh Prakash Nirmal, Mahidol University for revising the English language of this manuscript.

Contributors DB and MS conceptualised the study and prepared draft of study protocol. DB, MS, VW and CS developed study design, and planed of study. CS prepared draft of manuscript, DB and MS critically reviewed draft version. Finally, all authors read and approved the final manuscript.

Funding The authors have not declared a specific grant for this research from any funding agency in the public, commercial or not-for-profit sectors.

Competing interests None declared.

Patient consent for publication Not applicable.

Provenance and peer review Not commissioned; externally peer reviewed.

Supplemental material This content has been supplied by the author(s). It has not been vetted by BMJ Publishing Group Limited (BMJ) and may not have been peer-reviewed. Any opinions or recommendations discussed are solely those of the author(s) and are not endorsed by BMJ. BMJ disclaims all liability and responsibility arising from any reliance placed on the content. Where the content includes any translated material, BMJ does not warrant the accuracy and reliability of the translations (including but not limited to local regulations, clinical guidelines, terminology, drug names and drug dosages), and is not responsible for any error and/or omissions arising from translation and adaptation or otherwise.

Open access This is an open access article distributed in accordance with the Creative Commons Attribution Non Commercial (CC BY-NC 4.0) license, which permits others to distribute, remix, adapt, build upon this work non-commercially, and license their derivative works on different terms, provided the original work is properly cited, appropriate credit is given, any changes made indicated, and the use is non-commercial. See: http://creativecommons.org/licenses/by-nc/4.0/.

ORCID iD

Daniel Behrendt http://orcid.org/0000-0001-5559-2814

\section{REFERENCES}

1 Derek W. Securing good care for older people: taking a long-term view. London: King's Fund, 2006: 345. www.kingsfund.org.uk/ publications/securing-good-care-older-people

2 Schanze JL, Zins S. Undercoverage of the elderly institutionalized population: the risk of biased estimates and the potentials of weighting. Surv Methods Insights Field2019;19.

3 Drageset J, Espehaug B, Hallberg IR, et al. Sense of coherence among cognitively intact nursing home residents--a five-year longitudinal study. Aging Ment Health 2014;18:889-96.

4 World Health Organization. Global strategy and action plan on ageing and health. Geneva: World Health Organization, 2017. https://www. who.int/ageing/WHO-GSAP-2017.pdf?ua $=1$

5 Miller CA. Diverse health care settings for older adults. In: Miller CA, ed. Nursing for wellness in older adults. 6th edn. Philadelphia: Lippincott Williams \& Wilkins, 2012: 75-93.

6 Sanford AM, Orrell M, Tolson D, et al. An international definition for "nursing home". J Am Med Dir Assoc 2015;16:181-4.

7 Luppa M, Luck T, Matschinger H. Predictors of nursing home admission of individuals without a dementia diagnosis before admission - results from the Leipzig Longitudinal Study of the Aged (LEILA 75+). BMC Health Serv Res 2010;29:186.

8 Holup AA, Hyer K, Meng $\mathrm{H}$, et al. Profile of nursing home residents admitted directly from home. J Am Med Dir Assoc 2017;18:131-7.

9 Braunseis F, Deutsch T, Frese T, et al. The risk for nursing home admission (NHA) did not change in ten years--a prospective cohort study with five-year follow-up. Arch Gerontol Geriatr 2012;54:e63-7.

10 Drageset J, Dysvik E, Espehaug B, et al. Suffering and mental health among older people living in nursing homes-a mixed-methods study. PeerJ 2015;3:e1120.

11 O'Caoimh R, O'Donovan MR, Monahan MP, et al. Psychosocial impact of COVID-19 nursing home restrictions on visitors of residents with cognitive impairment: a cross-sectional study as part 
of the engaging remotely in care (ERiC) project. Front Psychiatry 2020;11:585373.

12 Brownie S, Horstmanshof L, Garbutt R. Factors that impact residents' transition and psychological adjustment to long-term aged care: a systematic literature review. Int J Nurs Stud 2014;51:1654-66.

13 Behrendt D, Schirmer M, Werner N. Etikette oder innovation? Eine Bestandsaufnahme sozialer Betreuung in stationären Pflegeeinrichtungen. NOVAcura 2020;51:25-8.

14 World Health Organization Regional Office for Europe (DK). Health 2020: social protection and health. Copenhagen: World Health Organization, 2015: 8. https:/www.euro.who.int/_data/assets/pdf file/0019/324631/Health-2020-Social-protection-and-health-en.pdf

15 Xue R, Gepp A, O'Neill TJ, et al. Financial literacy amongst elderly Australians. Account Finance 2018;59(Suppl 1):887-918.

16 van den Brink AMA, Gerritsen DL, de Valk MMH, et al. What do nursing home residents with mental-physical multimorbidity need and who actually knows this? A cross-sectional cohort study. Int $J$ Nurs Stud 2018;81:89-97.

17 Coimbra VdaSA, Silva RMCRA, Joaquim FL, et al. Gerontological contributions to the care of elderly people in long-term care facilities. Rev Bras Enferm 2018;71:912-9.

18 Barker RO, Craig D, Spiers G, et al. Who should deliver primary care in long-term care facilities to optimize resident outcomes? A systematic review. J Am Med Dir Assoc 2018;19:1069-79.

19 Haaster F. Soziale Betreuung in stationären: Pflegeeinrichtungen-Ein Aufgabenfeld der Sozialarbeit. Norderstedt: GRINN Verlag, 2005: 21. ISBN: 978-3-638-93930-0.

20 Michel J-P, Ecarnot F. The shortage of skilled workers in Europe: its impact on geriatric medicine. Eur Geriatr Med 2020;11:345-7.

21 Eastwood JB, Conroy RE, Naicker S, et al. Loss of health professionals from sub-Saharan Africa: the pivotal role of the UK. Lancet 2005;365:1893-900.

22 Wu Q, Zhao L, Ye X-C. Shortage of healthcare professionals in China. BMJ 2016;354:i4860.

23 Pilisuk M. Delivery of social support: the social inoculation. Am J Orthopsychiatry 1982;52:20-31.

24 Cohen S, Gottlieb BH, Underwood LG. Social relationships and health. In: Cohen S, Underwood LG, Gottlieb BH, eds. Social support measurement and intervention: a guide for health and social scientists. New York: Oxford University Press, 2000: 3-25.

$25 \mathrm{Ma} \mathrm{L}, \mathrm{Li}$ Y, Wang J, et al. Quality of life is related to social support in elderly osteoporosis patients in a Chinese population. PLoS One 2015;10:e0127849.

26 LaRocca MA, Scogin FR. The effect of social support on quality of life in older adults receiving cognitive behavioral therapy. Clin Gerontol 2015;38:131-48.

27 Uchino BN. Social support and health: a review of physiological processes potentially underlying links to disease outcomes. J Behav Med 2006;29:377-87.

28 Turner JR, Brown RL. Social support and mental health. In: Scheid TL, Brown TN, eds. Social contexts, theories, and system. 2nd ed. Cambridge University Press, 2010: 200-12. ISBN: -13 978-0-52172891-1.

29 Nair P, Gill JS, Sulaiman AH, et al. Mental health correlates among older persons residing in Malaysian nursing homes during the COVID-19 pandemic. Asia Pac J Public Health 2021;33:940-4.

30 Wang H-H, Wu S-Z, Liu Y-Y. Association between social support and health outcomes: a meta-analysis. Kaohsiung J Med Sci 2003; $19: 345-50$

31 Iglesias S, Arias AV. Structural and functional social support in elderly objective and subjective health ratings. Eur J Investig Health Psychol Educ 2015;5:243-52.

32 Veiel HOF. Dimensions of social support: a conceptual framework for research. Soc Psychiatry 1985;20:156-62.

33 Wills TA, Shinar O. Measuring perceived and received social support. In: Cohen S, Underwood LG, Gottlieb BH, eds. Social support measurement and intervention: a guide for health and social scientists. New York: Oxford University Press, 2000: 86-135.

34 Mikkelsen ASB, Petersen S, Dragsted AC, et al. Social interventions targeting social relations among older people at nursing homes: a qualitative synthesized systematic review. Inquiry 2019;56:46958018823929.

35 Testad I, Corbett A, Aarsland D, et al. The value of personalized psychosocial interventions to address behavioral and psychological symptoms in people with dementia living in care home settings: a systematic review. Int Psychogeriatr 2014;26:1083-98.

36 Wenborn J. Meaningful activities. In: Lohrmann SS, ed. Dementia in nursing homes. Cham: Springer International Publishing AG, 2017: 5-20.

37 Callaghan P, Morrissey J. Social support and health: a review. J Adv Nurs 1993;18:203-10.

38 Hendryx M, Green CA, Perrin NA. Social support, activities, and recovery from serious mental illness: STARS study findings. J Behav Health Serv Res 2009;36:320-9.

39 Arksey H, O'Malley L. Scoping studies: towards a methodological framework. Int J Soc Res Methodol 2005;8:19-32.

40 Peters MD, Godfrey C, Mclnerney P. Scoping reviews (2020 version). In: Aromataris E, Munn Z, eds. JBI manual for evidence synthesis. JBI, 2020. https://synthesismanual.jbi.global

41 Lockwood C, Dos Santos KB, Pap R. Practical guidance for knowledge synthesis: Scoping review methods. Asian Nurs Res 2019:13:287-94.

42 Daudt HML, van Mossel C, Scott SJ. Enhancing the scoping study methodology: a large, inter-professional team's experience with Arksey and O'Malley's framework. BMC Med Res Methodol 2013;13:48.

43 Bramer WM, Giustini D, Kleijnen J, et al. Searching Embase and MEDLINE by using only major descriptors or title and abstract fields: a prospective exploratory study. Syst Rev 2018;7:200.

44 Tricco AC, Lillie E, Zarin W, et al. PRISMA extension for scoping reviews (PRISMA-ScR): checklist and explanation. Ann Intern Med 2018;169:467-73.

45 Moher D, Liberati A, Tetzlaff J, et al. Preferred reporting items for systematic reviews and meta-analyses: the PRISMA statement. PLoS Med 2009;6:e1000097.

46 Peters MDJ, Marnie C, Tricco AC, et al. Updated methodological guidance for the conduct of scoping reviews. JBI Evid Synth 2020;18:2119-26.

47 Joanna Briggs Institute. The Joanna Briggs Institute Reviewers' Manual 2015 Methodology for JBI Scoping Reviews. 24. South Australia: The Joanna Briggs Institute, 2015.

48 Hong QN, Fàbregues S, Bartlett G, et al. The mixed methods appraisal tool (MMAT) version 2018 for information professionals and researchers. Educ Inform 2018;34:285-91.

49 Hong QN, Gonzalez-Reyes A, Pluye P. Improving the usefulness of a tool for appraising the quality of qualitative, quantitative and mixed methods studies, the mixed methods appraisal tool (MMAT). J Eval Clin Pract 2018;24:459-67.

50 Hong QN, Pluye P, Fàbregues S, et al. Improving the content validity of the mixed methods appraisal tool: a modified e-Delphi study. J Clin Epidemiol 2019;111:49-59.

51 Pérez-Zepeda MU, García-Peña C, Gutiérrez-Robledo LM. The need for differentiated research methodology in aging. In: García-Peña C, Gutiérrez-Robledo LM, Pérez-Zepeda MU, eds. Aging research - methodological issues. 2nd ed. Cham: Springer International Publishing, 2018: 1-9.

52 World Health Organization. Integrated care for older people: guidelines on community-level interventions to manage declines in intrinsic capacity. Geneva: World Health Organization, 2017: 46. https://apps.who.int/iris/handle/10665/258981 